\title{
Wire Up on Carbon Nanostructures! How To Play a Winning Game
}

Silvia Marchesan,* Michele Melchionna,* and Maurizio Prato*

Center of Excellence for Nanostructured Materials, INSTM, Unit of Trieste, Dipartimento di Scienze Chimiche e Farmaceutiche, University of Trieste, Piazzale Europa 1, 34127 Trieste, Italy

\section{ABSTRACT}

Carbon nanotubes and graphene possess a unique extended $\pi$-system that makes them stand out among carbon nanostructures. The resulting electronic properties enable electron or charge flow along one or two directions, respectively, thus offering the opportunity to connect electronically different entities that come into contact, be they living cells or catalytic systems. Using these carbon nanostructures thus holds great promise in providing innovative solutions to address key challenges in the fields of medicine and energy. Here, we discuss how chemical functionalization of these carbon nanostructures is a crucial tool to master their properties and deliver innovation.

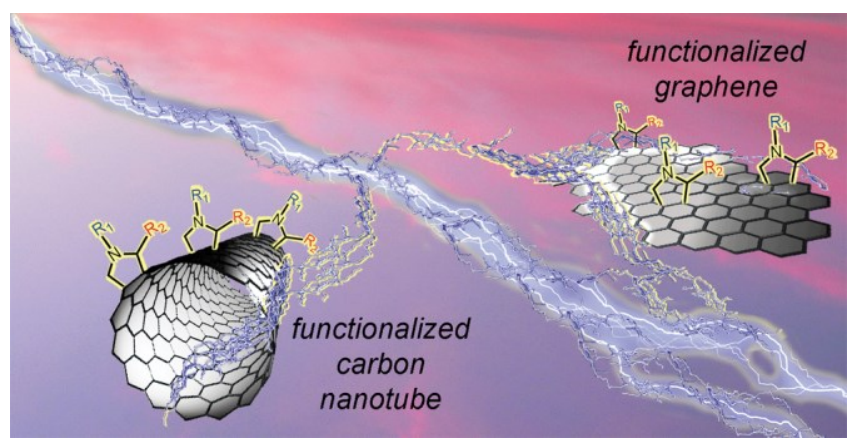

The nanotechnology revolution has begun, and is set to advance our world beyond our collective imagination. The target is to provide innovative answers to well-known issues that span all aspects of our everyday life. Can we grow older and healthier? Can we better exploit solar light to set ourselves free from fossil fuel dependence? These questions may seem so disparate as to have nothing in common. Actually, carbon nanostructures (CNS) may provide just the right connection. Over the last two decades, carbon nanotubes (CNTs) and, more recently, graphene (G), have proven to possess the versatility to be leading figures in different areas of research, including nanoelectronics and photovoltaics, ${ }^{1}$ electrochemical energy storage devices, ${ }^{2}$ biosensing, ${ }^{3}$ optoacustic imaging, ${ }^{4}$ on-demand drug delivery, ${ }^{5}$ and tissue engineering. ${ }^{6}$ Significant scientific advances have been possible thanks to the remarkable features of CNS, including robustness, lightness, elasticity, conductivity, and the ability to respond to light, just to name a few. Importantly, CNS can also be deposited on substrates to create an active coating that interfaces the underlying material with catalysts, solutions, cells, or light. 
Carbon nanostructures come in different sizes and shapes, but CNTs and G stand out for their unique network of $s p^{2}$ carbon atoms. Their extended $\pi$-electron structure and their distinctive geometry at the nanoscale offer an ideal resource for the inventive scientist and a perfect playground for the creative chemist. The ability of carbon to interact and to react with a wide variety of chemical elements offers a great advantage over inorganics, although stability in organics can be an issue. Importantly, the extended conjugated $\pi$-system enables electron or charge flow along one or two directions, depending on whether CNTs or G are used, respectively. As a result, it is possible to connect different functional components electronically, be they living cells or catalytic systems. Effectively, CNTs and G molecularly and electronically bridge in a manner that is useful across many disciplines of science, from medicine to energy.

In reality, these innovative structures come with their own set of challenges, i.e., the well-known tendency toward aggregation and safety concerns regarding large-scale use. In fact, they are seldom used in their pristine states, and an almost inevitable step is their chemical functionalization prior to use. ${ }^{7}$ It is in this arena that the organic chemist has to find the right strategy to tame the nanomaterials to play a winning game. Covalent functionalization indeed provides a valid approach to address such issues by rendering CNTs or G more amenable to industrial processing, liquid phase-dispersibility, and even biological use. ${ }^{8}$ Their sizes and shapes can be fine-tuned, for instance by oxidative shortening or longitudinal cutting of $\mathrm{CNTs},{ }^{9}$ according to the desired application. Together with the introduction of polar groups, this is also an effective strategy to decrease their tendency toward aggregation, thus achieving good water dispersibility, and to lift completely the pathogenicity that is associated with asbestos-like aggregates. ${ }^{10}$

Today, the tools available to functionalize CNS are widely varied and well-established, but we note that we are leveraging the results of over 20 years of research in this field. When $\mathrm{C}_{60}$ fullerene, the first member of the CNS family, was discovered in 1985, it was a puzzle to establish its chemical structure, and solving that puzzle was worth a Nobel prize. ${ }^{11}$ Scientists were left mesmerized by the "soccer ball" geometry, and it took a while to understand and to master its chemical reactivity. At present, among the many options available for covalent functionalization of CNS, a general and useful approach is the 1,3dipolar cycloaddition of azomethine ylides, which can be generated from a broad number of precursors, including aldehydes and amino acids, or aziridines. ${ }^{12}$ The approach is versatile as it enables the introduction of a variety of $N$ - and $C$-substitutions onto a newly installed pyrrolidine ring (Fig. 1), therefore creating the possibility of increasing the polarity of the highly hydrophobic carbon core to achieve water solubility. ${ }^{13}$ The same concept can also be extended to carbon onions ${ }^{14}$ and nanohorns, ${ }^{15}$ CNTs, ${ }^{16}$ and G. ${ }^{17}$ The approach can be induced by microwave irradiation, ${ }^{18}$ and can occur also in ionic liquids ${ }^{19}$ or solvent-free conditions, and in a reversible manner. ${ }^{20}$ The use of cycloaddition reactions also has the net advantage of permitting bulk functionalization of $G$ sheets, while other approaches functionalize only its edges. ${ }^{21}$ Moreover, the presence of additional groups harnesses these CNS for post-modification with more complex molecular entities, ${ }^{22}$ enabling viability of diverse processes, such as catalysis. ${ }^{23}$ Functionalization enables CNS processing, such as dispersion for electrospinning ${ }^{24}$ or the production of composite materials. ${ }^{25}$ It can occur in dispersions ${ }^{26}$ or on solid substrates, which can then be exploited to affect the outcome of functionalization. ${ }^{27}$ Mastering the chemical reactivity of CNTs and 
$\mathrm{G}$ toward the desired application is thus a fundamental step to exploit their extraordinary properties fully and to bring innovation to a range of scientific fields.
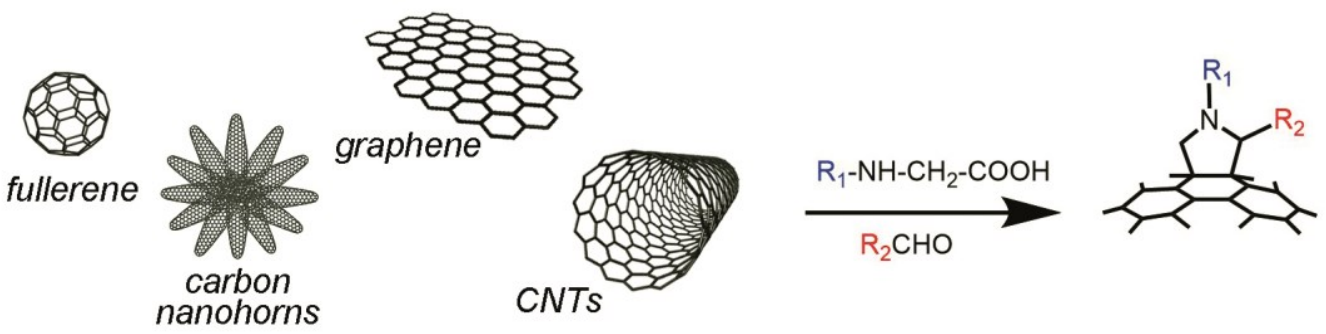

Fig. 1. Several carbon nanostructures (CNS) can be covalently functionalized by means of 1,3-dipolar cycloaddition, which offers the possibility of introducing $\mathrm{N}$ - and C-substitutions on a newly installed pyrrolidine ring.

\section{Medicinal Chemistry Applications}

In medicine, one of the most challenging objectives is the tissue engineering and regeneration of the brain and the heart. Their structural and functional complexity can be overwhelming to reproduce by means of an artificial nanomaterial scaffold. However, it may be wise to dissect the problem to look first at the simplest functional elements, i.e., the electrically conductive network of specialized cells. Which nanomaterial could provide the appropriate substrate to wire up disconnected units? Carbon is by definition the heart of organic chemistry; thus, it would seem appropriate to have it as a core element to reconnect living entities. In addition, CNTs and G offer a rich inventory in terms of geometry, size, reactivity, and properties. Carbon nanotubes stand out as structural candidates to mimic the elongated heart myofibrils or to guide neuronal axons. Their robust yet light and flexible nature, together with their high conductivity, are ideal properties from the point of view of physiological function. Indeed, various teams have combined the structural and functional fitness of CNTs to suit the stringent requirements for growing healthy networks of cardiomyocytes or neurons. It is not just a matter of providing an appropriate substrate for cell growth, it has actually emerged that CNTs have an electrifying effect on cells. ${ }^{28}$ In other words, specialized cells grown in contact with CNTs appear to be electrically more active.

In particular, cardiomyocytes grown on CNT-coated substrates display increased expression of gap junctions and, overall, a more mature phenotype, with enhanced electrophysiological behavior and implemented networking and maturation into functional syncytia (Fig. 2). ${ }^{29}$ Hydrogels containing CNTs constitute excellent grafts for functional integration with infarct myocardium, although signs of mild inflammation can be seen. Interestingly, their in vivo efficacy seems to be related to integrin-mediated up-regulation of adhesive and electrochemical junctions in cardiomyocytes. ${ }^{30}$ For heart tissue engineering, CNTs can also constitute a templating guide for the electrospinning of hybrid hydrogel nanofibers that form a superb biomimicking scaffold that is capable of matching the orientation index of the left ventricular tissue, thus promoting cardiomyocyte alignment and synchronous beating. ${ }^{31}$ Remarkably, thermoresponsive CNT-based hydrogels effectively improve stem-cell engraftment, survival, and differentiation in the infarcted heart, thus increasing their therapeutic efficacy for the 
treatment of myocardial infarction..$^{32}$ In addition, mastering their conductive properties leads to the creation of electroactive scaffolds that hold promise to solve arrhythmias as well. ${ }^{33}$ Graphene derivatives, including graphene oxide (GO), are equally leading candidates for heart tissue regeneration. Their unrivalled performance in the three-dimensional (3D) printing of an artificial heart holds promise for high innovation potential. ${ }^{34}$ For instance, 3D heart tissue containing living cells can be formed on a basal plane of a GO-bearing hydrogel through layer-by-layer deposition of alternate sheets of cells and adhesive GO-substrates, thus yielding a multilayer cardiac tissue that is able to beat and to pump. ${ }^{35}$

A

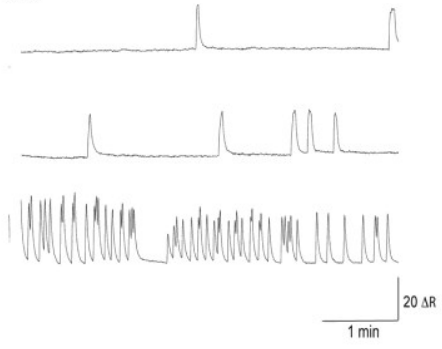

B

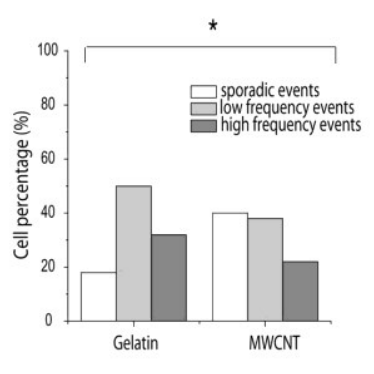

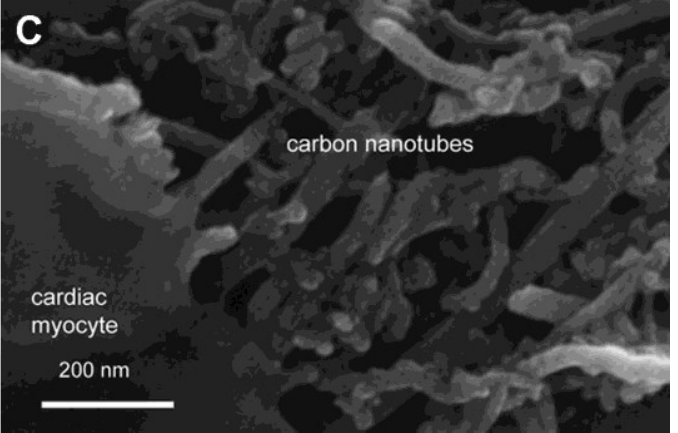

Fig. 2. A) Typical traces for calcium oscillations that exemplify (top) sporadic events, (center) low frequency events and(bottom) high frequency events in oscillating cardiomyocytes. B) Cells grown on CNTs display a significantly higher number of sporadic calcium oscillations relative to gelatin control. C) Scanning electron microscope image showing a cardiomyocyte in close contact with CNTs. Reprinted from ref. 29. Copyright 2013 American Chemical Society.

In the field of nerve tissue engineering and artificial neural networks, ${ }^{36}$ CNTs and $\mathrm{G}$ could bring innovative 3D scaffolds, ${ }^{37}$ thanks to a boosting effect on neuron activity ${ }^{38}$ and their capability to modulate the immune response. ${ }^{39}$ Carbon nanotubes can have the dual function of being templates for the hierarchical alignment of different components into nanofibrous biomaterials, and of being electrical actuators of neural differentiation of stem cells when they are appropriately embedded in nanostructured biocomposites. ${ }^{40}$ This is not surprising if we consider the established electrifying effect of interfacing CNTs with neurons, which anticipate the signs of functional maturation, including the generation of action potentials or voltage-dependent currents. ${ }^{38}$ Remarkably, a fortifying effect on neurite outgrowth and synaptic inputs is also observed on spinal slices as a more complex model of multilayered tissue. ${ }^{41}$ It has been proposed that such an effect is the result of electrical "shortcuts" permitted by CNTs in direct contact with cell membranes to connect proximal and distal compartments of the neuron (Fig. 3). ${ }^{42}$ However, it is crucial to select the route to functionalize CNTs for their application in tissue engineering carefully. The importance of the level and nature of covalent modification is just now starting to emerge. For instance, amino-functionalized CNTs produced by different methods and of dissimilar lengths can lead to disparate outcomes of inflammation response in the brain. ${ }^{43}$ Use of invasive radical protocols that easily enable high functionalization levels can affect the metallic structure of the CNT sidewalls, thus resulting in high resistance and loss of the boosting effect on neuronal activity. ${ }^{44}$ Instead, it may be preferable to adopt a more controlled approach, leading to a better compromise between the extent of added functional groups and the preservation of the graphitic structure, thus maintaining the CNT physico-chemical properties required to stimulate 
neuronal networks at the interface. ${ }^{44}$ Such careful selection of the functionalization route can have important implications beyond the field of tissue regeneration as well, including the fabrication of microelectrodes to study chemical and electrical neuronal signalling, ${ }^{45}$ and, more generally, the biointegration of chronic implants ${ }^{46}$ or other microdevices to promote local repair at the site of nerve injury. ${ }^{47}$
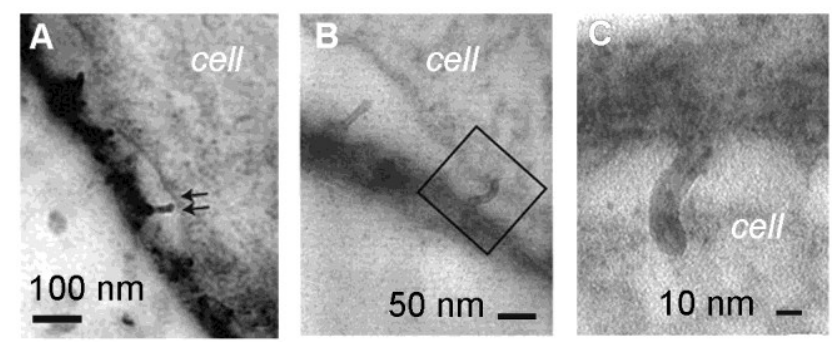

Fig. 3. A-C) Low-to-high magnification transmission electron microscopy images of sagittal sections showing the direct contact between a neuron cell (top and right portions of images $A-B$, and bottom of C) and an underlying CNT-coating (darker section line), with CNTs piercing the cell membrane (black arrows in $A$, black square in $B$, and C). Reprinted with permission from ref. 42. Copyright 2008 Nature Publishing Group.

Graphene is also an ideal substrate for cell adhesion, as it enables optimal cytoskeleton anchoring by sustaining lateral stress and providing the appropriate level of tension. ${ }^{48}$ This finding is consistent with the reported role of integrin-mediated activation of mechanotransduction pathways that lead to the differentiation of neural stem cells grown on a hybrid nanofibrous scaffold containing G. ${ }^{49}$ This nanomaterial and its derivatives can also act as a pre-concentrating agent of biochemical inducers that adsorb on its surface. ${ }^{50}$ This fact can easily be rationalized considering that it is well known that graphitic surfaces can establish strong interactions with proteins, although fine control is imperative to preserve protein function fully. ${ }^{51}$ As an example of applied molecular adsorption to induce cell adhesion, use of poly-D-lysine-coatings with micropatterned $G$ yields artificial neural networks that reproduce tailored designs. While polylysine initially adsorbs all over the surface, resulting in random cell attachment, over time, the polypeptide forms a thicker layer on $G$, and eventually affects neural cell movement, which becomes directed toward G-coated areas. ${ }^{52}$ As an alternative to polypeptides, amine functionalities can also be appended through covalent functionalization of G or GO. Such nanomaterial components can form hybrids that are able to inhibit bacterial biofilm formation, and that may thus provide useful coatings for biomedical implants. ${ }^{53}$ It would be interesting to explore the effects of chemical modification of $G$ nanomaterials for tissue engineering applications, especially considering the high potential already shown by $\mathrm{G}$ despite its infancy in the field. We anticipate that, analogously to CNTs, routes affecting $\mathrm{G} \pi$-structure will, to a different extent, determine the changes in its electronic properties, and therefore its influence on cells; the two-dimensional geometry and dissimilar curvature of $\mathrm{G}$ relative to nanotubes will provide interesting additional factors to determine cell fate.

\section{Energy Applications}

Energy is another field that has enjoyed great benefits from CNTs and G. These CNS have been combined with inorganic catalysts, particularly metal oxides $\left(\mathrm{MO}_{\mathrm{x}}\right)$ and nanoparticles $(\mathrm{NP})$, in countless 
numbers of nanocomposites and hybrids. In general, catalysis by these nanomaterials has been exploited for almost 30 years, and overarches several classes of reactions. Energy is an emerging field of research that is gaining a strong momentum, in parallel with the general interest in new and efficient processes that promise to set our society free from our unsustainable thirst for fossil fuels. The benefits of integrating a CNS component into a catalyst design are evident. Above all, it may be key to have an efficient interface between the organic and inorganic phases so as to produce electronic crosstalk. ${ }^{54}$ In some cases, such crosstalk is manifested with the formation of heterojunctions or Schottky barriers that are at the core of the catalytic performance enhancement. It has also been hypothesized that the (semi)conductive scaffold of the CNS can act as an efficient electron sink, thus influencing factors that are important for the particular catalysis, such as charge-recombination rates (photocatalysis) or charge mobility (electrocatalysis). Overall, the assembly of high-performing catalysts relies on well-designed engineering of the CNS, with carefully chosen synthetic steps. One key factor is the functionalization of CNTs or G prior to hybridization with the inorganic precursor. This step is required to equip the CNS with anchor points for a firm linkage with the inorganic component.

There are two possible functionalization strategies, one covalent and one supramolecular, and each strategy has its own pros and cons. On one hand, covalent functionalization enables robust binding of the metal catalyst. However, this method also implies that the $\mathrm{sp}^{2} \pi$-conjugated systems of the CNS are partially disrupted, resulting in expected decreases in the electronic performance. On the other hand, CNS electronic features can be fully preserved by means of non-covalent routes, typically by simply adsorbing ligand or linker molecules onto the CNS via $\pi$-stacking or van der Waals interactions. The downside in this case is overall weaker binding of the catalysts. As a result, desorption can occur at later stages when experimental conditions change, for instance during catalysis, leading to poorer interactions between the metal and the CNS. How can one make correct choices for CNS functionalization? Unfortunately, this question is not trivial, as there is no universal solution. One must consider the specific process of interest, the operative conditions, and which particular catalytic aspect is being investigated. Once CNTs or G have been appropriately functionalized, interfacing with the inorganic phase can be performed through various strategies, and, in general, they are designed to have maximum contact between the two phases.

One well-suited example is the assembly of a hybrid system based on oxidized multi-wall CNTs interfaced with core-shell NPs of noble metal $(\mathrm{M})$ surrounded by metal oxide $\left(\mathrm{MO}_{\mathrm{x}}\right)$, i.e., $\mathrm{M} @ \mathrm{MO} \mathrm{x}_{\mathrm{x}}$. The synthesis is performed via a sol-gel approach and is flexible enough to modulate the composition, and to include $\mathrm{Pd}$ or $\mathrm{Pt}$ as $\mathrm{M}$, and $\mathrm{TiO}_{2}, \mathrm{CeO}_{2}$, and $\mathrm{ZrO}_{2}$ as $\mathrm{MO}_{\mathrm{x}}$. A key factor for the successful assembly of this architecture is the pre-oxidation of the CNTs for the double function of 1 ) improving CNS dispersibility into the solvent media and 2) generating a reasonable amount of $-\mathrm{COOH}$ and $-\mathrm{OH}$ groups to bind the metal precursor alkoxide shell via ligand exchange. The synthetic strategy is very powerful as it allows a balanced hierarchy of the three components, with the M and the CNT mutually interacting while being embedded separately into the $\mathrm{MO}_{x}$ matrix (Fig. 4). 

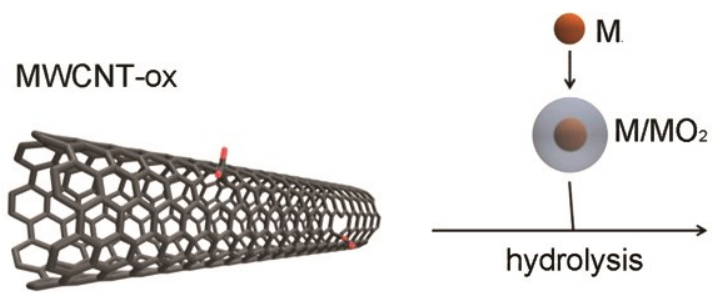

MWCNT@M/MO2

hydrolysis
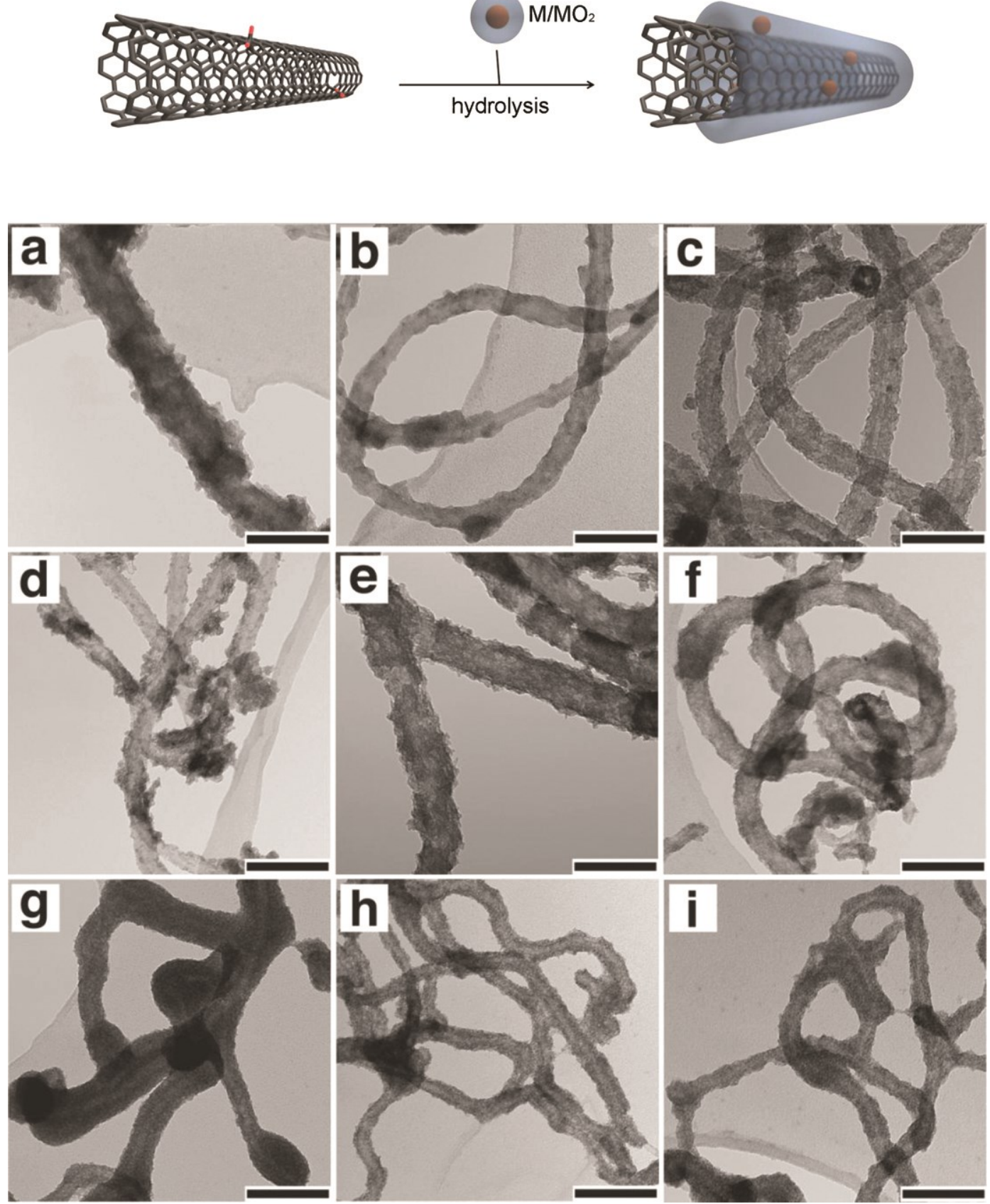

Fig. 4. Top. Synthetic scheme showing assembly of organic/inorganic hybrid catalyst (right) based on oxidized CNTs (left, with $\mathrm{COOH}$ groups in red) and inorganic NPs (center) based on a noble metal core ( $\mathrm{M}$, in orange) and a metal oxide shell $\left(\mathrm{MO}_{2}\right.$, in light grey). Bottom. Representative TEM images of MWCNTs@(1 wt \%)Pd-oxide nanocomposites with (a-c) $\mathrm{TiO}_{2}$, (d-f) $\mathrm{ZrO}_{2}$, and (g-i) $\mathrm{CeO}_{2}$ shells. The weight concentration of MWCNTs is $(g) 5 \%,(a, d, h) 10 \%,(b, e, i) 15 \%$, and (c, f) $20 \%$. All scale bars represent $100 \mathrm{~nm}$. Adapted from ref 55. Copyright 2012 American Chemical Society. 
Among other reactions, the catalyst exhibits excellent performance in $\mathrm{H}_{2}$ production via methanol photoreforming, with the notable highest rates of $10 \mathrm{mmol} \cdot \mathrm{g}^{-1} \cdot \mathrm{h}^{-1}$ if $\mathrm{TiO}_{2}$ is used as the oxide shell and $\mathrm{Pt}$ as the core. Moreover, if the composition is switched to the $\mathrm{Pd@CeO}$ module, the catalyst presents excellent activity and stability for the low-temperature water-gas shift reaction. This latter example is another fundamental energetic process that enables the purification of $\mathrm{H}_{2}$ from $\mathrm{CO}$ impurities formed during conventional thermal reforming, while increasing $\mathrm{H}_{2}$ production. ${ }^{55}$ This example not only illustrates the great potential for exploiting CNTs in the field of energy, but it also highlights the structural complexity of successful materials, and the demanding synthetic design that they require. Moreover, the catalyst complexity is also linked to an intricate mechanism, which is indeed difficult to completely unveil. As new theoretical insights become available, and suitable mechanistic experiments are performed, the key factors dominating catalytic behavior will become clearer. The target is to master the tuning of CNS physicochemical properties for integration into more complex catalytic architectures to revolutionize energy conversion. In fact, regardless of the doubts as to the mechanism, and given the projected dominant role of $\mathrm{H}_{2}$ as an environmentally friendly fuel vector of the future, $\mathrm{H}_{2}$ photoproduction is enjoying great popularity. New, refined systems are reported daily, with activity and stability steadily increasing. At this pace of development, and in parallel with the enormous advances in the scaled-up preparation of high-quality CNS, we anticipate a new generation of solar fuel cells with CNS as essential components.

At present, one of the key challenges for using solar fuels is the low efficiency of most catalytic materials under visible light. In this scenario, the ad hoc tuning of CNS light absorption via chemical modification opens the tantalizing but not unrealistic vision of a fully sunlight-driven energy economy. Indeed, modification of photoelectrode surfaces with CNTs has proven to hold great potential. The concept is general enough to be extended to other CNS, for instance carbon nanohorns (CNHs), which are becoming increasingly popular. Their peculiar architecture offers the advantage of possessing notably high surface areas. As an example of applications, fluorine-doped tin oxide (FTO) electrodes modified with interlayers of $\mathrm{CNHs}$ can be covered with solar-sensitive $\mathrm{TiO}_{2}$ via spincoating for fine control over film thickness (Fig. 5). In this system, the pristine nanohorns create an electronic bridge that channels photoexcited electrons from $\mathrm{TiO}_{2}$ onto the FTO electrode. This component is an alternative for dyesensitized solar cells (DSSC) to replace standard $\mathrm{TiCl}_{4}$ pre-treatment. $^{56}$

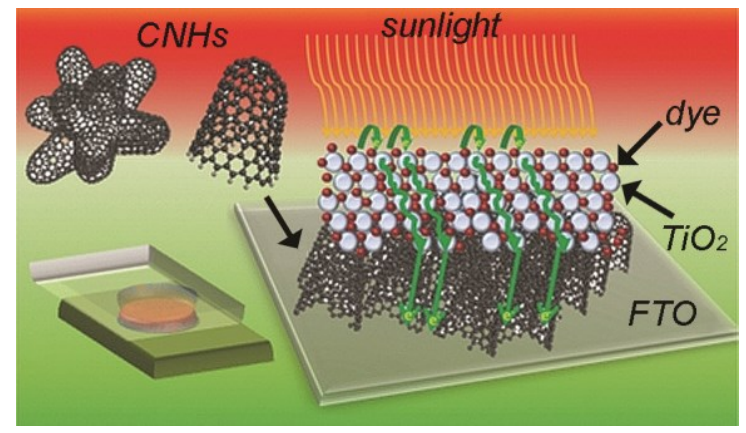

Fig. 5. Dye-sensitized solar cells (DSSC) prepared with carbon nanohorns show promise for sunlightdriven energy conversion. Reprinted with permission from ref. 56. Copyright 2014 Wiley-VCH. 
As an alternative to the photo-induced process, pure $\mathrm{H}_{2}$ electrocatalytic production is a shorter-term prospect, which also relies on the exceptional electronic properties of CNTs or G. Recent pioneering work revealed a strong electronic coupling between $\mathrm{MoS}_{2}$ and reduced graphene oxide ( $\mathrm{GGO}$ ) which was responsible for enhanced electrocatalytic hydrogen evolution (HER). The authors found much lower impedance for the G-based composite relative to free $\mathrm{MoS}_{2}$, highlighting the important role in chargecarrier mobility of the CNS support. ${ }^{57}$

If the $\pi$-conjugation of the CNS is to be left unperturbed, non-covalent attachment of catalytically active metals may be a more convenient choice. As one representative example, equipment of a bioinspired $\mathrm{Ni}$ bisdiphosphine complex with a pendant pyrene moiety permitted attachment to CNT sidewalls. This system proved to be an exceptional electrocatalyst for $\mathrm{H}_{2}$ evolution, with overpotential as small as $78 \mathrm{mV}$ and high current density. ${ }^{58} \mathrm{~A}$ similar concept was adopted for the modification of an indium tin oxide (ITO) electrode with a CNT-derived Ru complex, where the Ru catalyst was fixed by means of pyrene units. The resulting modified electrode could perform the kinetically challenging reaction of water oxidation, with the catalyst exhibiting turnover frequencies (TOFs) as high as $1700 \mathrm{cycles} \cdot \mathrm{h}^{-1} .{ }^{59}$

This last example introduces another exciting perspective in energy research, namely the fascinating possibility of carrying out artificial photosynthesis. At the heart of this natural phenomenon lies the energetically demanding water-oxidation process, carried out by a metal-oxygen cluster with four manganese atoms and one calcium atom, $\mathrm{Mn}_{4} \mathrm{Ca} .{ }^{60}$ Massive research is being conducted to find alternative catalysts that can perform water oxidation with high efficiency. Can CNTs or G hold the key to open this golden gate? Indeed, encouraging findings are bringing us closer to the dream goal of artificial photosynthesis. Most notably, a nanocatalyst based on the combination of a Ru polyoxometalate $\left(\mathrm{Ru}_{4} \mathrm{POM}\right)$ and functionalized $\mathrm{CNTs}$ was used to modify an oxygen-evolving anode. Also in this case, chemical functionalization of the CNS played a crucial role and was carefully designed. In particular, CNTs were first modified with charged dendrimers (polyamidoamine (PAMAM) ammonium), and then attached to the inorganic domain through electrostatic interactions using $\mathrm{Li}_{10} \mathrm{Ru}_{4} \mathrm{POM}$ derivative (Fig. 6). The so-modified ITO electrode exhibited TOF values for water oxidation as high as 306 $\mathrm{h}^{-1}$ and an overpotential as small as $0.35 \mathrm{~V} .{ }^{61}$ Subsequently, a more facile method for CNT functionalization was developed, consisting of either covalent or non-covalent approaches to link ammonium trimethylammonium moieties to capture the $\mathrm{Ru}_{4} \mathrm{POM}$ complex electrostatically. The covalent protocol was assisted by microwave irradiation to insert amine groups onto the $\pi$-conjugated pattern, while the non-covalent method made use of pyrene units. ${ }^{62}$

Despite these exceptional results, there is still debate as to whether $G$ could be a more suitable nanostructure to fulfil the same task. When the dendrimer approach described above for CNTs was translated onto $G$, experimental evidence indicated that $G$ can even outperform CNTs, with one order of magnitude higher TOFs and overpotentials as low as $0.30 \mathrm{~V}$. As with the CNT analogous catalyst, a key aspect to consider is the control over $\mathrm{G}$ properties. The essential step of $\mathrm{G}$ functionalization was carried out via a less destructive protocol based on 1,3-dipolar cycloaddition, ${ }^{12}$ rather than more conventional but highly invasive oxidation. ${ }^{63}$ 


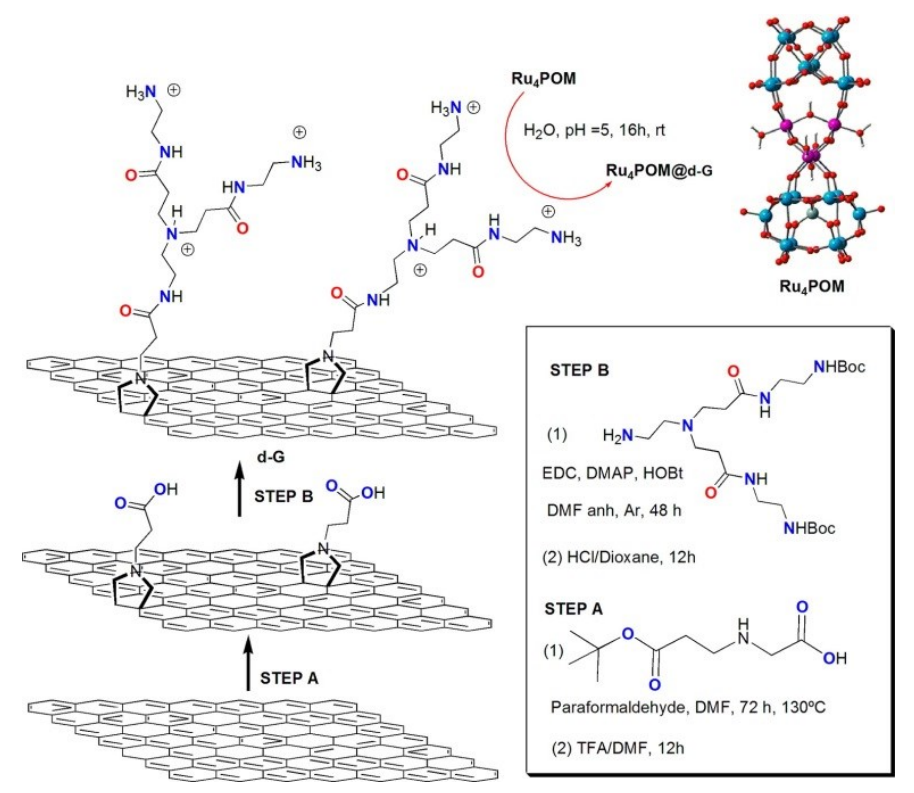

Fig. 6. Scheme showing the synthetic steps required for $\mathrm{G}$ derivatization with a nanocatalyst based on $\mathrm{Ru}$ polyoxometalate $\left(\mathrm{Ru}_{4} \mathrm{POM}\right)$ toward artificial photosynthesis. Reprinted from ref 63 Copyright 2013 American Chemical Society.

While photocatalysis by CNTs and G has immense promise in terms of supplying and storing energy with benign impacts on the environment, much work remains to improve efficiency. As an alternative, electrocatalysis appears to be within closer reach for short-to-medium term market breakthroughs. Indeed, CNS-modified electrodes are proliferating for a variety of other energy-related reactions. One such reaction, of huge importance for fuel cells, is the oxygen reduction reaction (ORR). This process is conventionally carried out by Pt catalysts supported on carbon, but it was shown to proceed with improved activity when the amorphous carbon support was replaced by $\mathrm{G}$, using FePt nanoparticles to decrease the required amount of costly and scarce Pt metal. ${ }^{64}$ Similar trends were observed with the employment of CNTs. ${ }^{65}$ There is a massive amount of research attempting to replace state-of-the-art Ptbased catalysts with cheaper metals, and both CNTs and $G$ are useful components to enhance performance. In one famous example, $\mathrm{CO}_{3} \mathrm{O}_{4}$ supported on $\mathrm{N}$-doped $\mathrm{GO}(\mathrm{N}$-GO) could be used for both ORR and the reverse oxygen evolution reaction (OER), displaying similar activity but higher stability than the corresponding Pt-based catalyst, ${ }^{66}$ while replacing $N$-GO with $N$-doped CNTs (N-CNTs) after hybridization with $\mathrm{CO}_{3} \mathrm{O}_{4}$ resulted in even higher ORR current densities. ${ }^{67}$ Another intriguing feature borne by CNTs as compared to $\mathrm{G}$ is the possibility of exploiting the inner cavity, endohedrally filling the tubes with several entities. This approach gives rise to what has been termed the "confinement effect", which leads to interesting and unusual characteristics. ${ }^{68}$ After preparation of a Fe-filled N-CNT electrocatalyst, it was suggested that across-layer electron transfer from endohedral Fe to the outer CNT layer could lead to efficient activity for ORR in acidic media. Other than directly being involved in the catalytic process, the CNTs also provide protection for the Fe from leaching under operation conditions. ${ }^{69}$ For the same hybrid, the external surface of the CNTs was carefully modified with benzoic acid groups in order to tune reactivity, and the catalyst was capable of carrying out both the ORR and the hydrogen evolution reaction (HER) at nearly neutral $\mathrm{pH}$. The importance of performing these two 
reactions under physiological conditions is a necessary condition for the development of microbial fuel cells, which are at the forefront of the energy revolution. ${ }^{70}$ In conclusion, CNS electronic benefits for catalysis are evident, and their chemical functionalization provides the right tool for fine control over the process. Informed choice over CNS derivatization will be key to make further quality progress in the energy field.

\section{CONCLUSIONS AND PROSPECTS}

The family of CNS has been growing steadily since 1985, providing interesting new tools for scientists every few years. In particular, those nanomaterials displaying extended $\pi$-conjugated systems, with CNTs and $\mathrm{G}$ as leaders, display intriguing electronic effects as discussed above. It took some time for researchers to gain familiarity with these nanomaterials and especially to understand that chemical functionalization is indispensable to handle and to apply these interesting CNS successfully. We are only now starting to understand better the deep implications of different chemical routes (e.g., radicalmediated, thermal cycloaddition, oxidation, etc.) for preservation and modulation of CNS electronic behavior. A fine balance between functionalization level, preservation of the $\pi$-system, and stability of the chemical species, while keeping their ability to respond to changes in the surrounding conditions is key. We have provided selected examples of a long research effort devoted to the use of CNS to bring about revolutions in energy and medicine. Mastering their unique properties offers tremendous opportunities to address the difficult challenges that our society is facing, and only continuous, longterm efforts will provide the right tools to enable full use of their unique bridging capabilities.

\section{ACKNOWLEDGMENTS}

Part of the work described in this Perspective was supported by the University of Trieste, the European Commission (ERC Advanced Grant CARBONANOBRIDGE No. 227135, project NMP4-SL-2012-310651 SACS, NMP4-SL-2012-310184 CARINHYPH, from the Italian Ministry of Education MIUR (cofin Prot. 2010N3T9M4 and FIRB Nanosolar RBAP11C58Y), and Consorzio Interuniversitario sui Materiali (INSTM).

\section{REFERENCES}

1. Campidelli, S.; Prato, M. Functionalization of Carbon Nanotubes for Nanoelectronic and Photovoltaic Applications. In Chemistry of Nanocarbons, John Wiley \& Sons, Ltd: 2010; pp 333-363.

2. Raccichini, R.; Varzi, A.; Passerini, S.; Scrosati, B. The Role of Graphene for Electrochemical Energy Storage. Nat. Mater. 2015, 14, 271-279.

3. Baptista, F. R.; Belhout, S. A.; Giordani, S.; Quinn, S. J. Recent Developments in Carbon Nanomaterial Sensors. Chem. Soc. Rev. 2015, 44, 4433-4453.

4. Taruttis, A.; Ntziachristos, V. Advances in Real-Time Multispectral Optoacoustic Imaging and its Applications. Nat. Photon. 2015, 9, 219-227.

5. Servant, A.; Leon, V.; Jasim, D.; Methven, L.; Limousin, P.; Fernandez-Pacheco, E. V.; Prato, M.; Kostarelos, K. Graphene-Based Electroresponsive Scaffolds as Polymeric Implants for On-Demand Drug Delivery. Adv. Healthc. Mater. 2014, 3, 1334-1343. 
6. Bosi, S.; Ballerini, L.; Prato, M., Carbon Nanotubes in Tissue Engineering. Top. Curr. Chem. 2014, 348, 181-204.

7. Melchionna, M.; Prato, M. Functionalizing Carbon Nanotubes: An Indispensible Step towards Applications. ECS J. Solid State Sci. Technol. 2013, 2, M3040-M3045.

8. Battigelli, A.; Menard-Moyon, C.; Da Ros, T.; Prato, M.; Bianco, A. Endowing Carbon Nanotubes with Biological and Biomedical Properties by Chemical Modifications. Adv. Drug Deliv. Rev. 2013, 65, 1899-1920.

9. Kosynkin, D. V.; Higginbotham, A. L.; Sinitskii, A.; Lomeda, J. R.; Dimiev, A.; Price, B. K.; Tour, J. M. Longitudinal Unzipping of Carbon Nanotubes To Form Graphene Nanoribbons. Nature 2009, 458, 872-876.

10. Ali-Boucetta, H.; Nunes, A.; Sainz, R.; Herrero, M. A.; Tian, B.; Prato, M.; Bianco, A.; Kostarelos, K. Asbestos-Like Pathogenicity of Long Carbon Nanotubes Alleviated by Chemical Functionalization.

Angew. Chem. Int. Ed. 2013, 52, 2274-2278.

11. Kroto, H. W.; Heath, J. R.; O'Brien, S. C.; Curl, R. F.; Smalley, R. E. C60: Buckminsterfullerene. Nature 1985, 318, 162-163.

12. Maggini, M.; Scorrano, G.; Prato, M. Addition of Azomethine Ylides to C60: Synthesis, Characterization, and Functionalization of Fullerene Pyrrolidines. J. Am. Chem. Soc. 1993, 115, 97989799.

13. Da Ros, T.; Prato, M.; Novello, F.; Maggini, M.; Banfi, E. Easy Access to Water Soluble Fullerene Derivatives via 1,3-Dipolar Cycloadditions of Azomethine Ylides to C60. J. Org. Chem. 1996, 61, 90709072.

14. Georgakilas, V.; Guldi, D. M.; Signorini, R.; Bozio, R.; Prato, M. Organic Functionalization and Optical Properties of Carbon Onions. J. Am. Chem. Soc. 2003, 125, 14268-14269.

15. Cioffi, C.; Campidelli, S.; Brunetti, F. G.; Meneghetti, M.; Prato, M. Functionalization of Carbon Nanohorns. Chem. Commun. 2006, 20, 2129-2131.

16. Georgakilas, V.; Kordatos, K.; Prato, M.; Guldi, D. M.; Holzinger, M.; Hirsch, A. Organic Functionalization of Carbon Nanotubes. J. Am. Chem. Soc. 2002, 124, 760-761.

17. Quintana, M.; Spyrou, K.; Grzelczak, M.; Browne, W. R.; Rudolf, P.; Prato, M. Functionalization of Graphene via 1,3-Dipolar Cycloaddition. ACS Nano 2010, 4, 3527-3533.

18. Brunetti, F. G.; Herrero, M. A.; Munoz, J. d. M.; Diaz-Ortiz, A.; Alfonsi, J.; Meneghetti, M.; Prato, M.; Vazquez, E. Microwave-Induced Multiple Functionalization of Carbon Nanotubes. J. Am. Chem. Soc. 2008, 130, 8094-8100.

19. Guryanov, I.; Toma, F. M.; Montellano Lopez, A.; Carraro, M.; Da Ros, T.; Angelini, G.; D'Aurizio, E.; Fontana, A.; Maggini, M.; Prato, M.; Bonchio, M. Microwave-Assisted Functionalization of Carbon Nanostructures in lonic Liquids. Chem. - Eur. J. 2009, 15, 12837-12845.

20. Brunetti, F. G.; Herrero, M. A.; De Munoz, J.; Giordani, S.; Diaz-Ortiz, A.; Filippone, S.; Ruaro, G.; Meneghetti, M.; Prato, M.; Vazquez, E. Reversible Microwave-Assisted Cycloaddition of Aziridines to Carbon Nanotubes. J. Am. Chem. Soc. 2007, 129, 14580-14581.

21. Quintana, M.; Montellano, A.; del Rio astillo, A. E.; Van endeloo, G.; Bittencourt, C.; Prato, M. Selective Organic Functionalization of Graphene Bulk or Graphene Edges. Chem. Commun. 2011, 47, 9330-9332.

22. Melchionna, M.; Prato, M. Functionalization of Carbon Nanotubes. In Nanocarbon-Inorganic Hybrids: Next Generation Composites for Sustainable Energy Applications. Eder, D.; Schlögl, R., Eds. Walter de Gruyter GmbH \& Co KG: 2014.

23. Melchionna, M.; Marchesan, S.; Prato, M.; Fornasiero, P. Carbon Nanotubes and Catalysis: The Many Facets of a Successful Marriage. Catal. Sci. Technol. 2015, 5, 3859-3875. 
24. Sui, X.-M.; Giordani, S.; Prato, M.; Wagner, H. D. Effect of Carbon Nanotube Surface Modification on Dispersion and Structural Properties of Electrospun Fibers. Appl. Phys. Lett. 2009, 95, 233113/1233113/3.

25. Georgakilas, V.; Bourlinos, A.; Gournis, D.; Tsoufis, T.; Trapalis, C.; Mateo-Alonso, A.; Prato, M. Multipurpose Organically Modified Carbon Nanotubes: From Functionalization to Nanotube Composites. J. Am. Chem. Soc. 2008, 130, 8733-8740.

26. Quintana, M.; Vazquez, E.; Prato, M. Organic Functionalization of Graphene in Dispersions. Acc. Chem. Res. 2013, 46, 138-148.

27. Criado, A.; Melchionna, M.; Marchesan, S.; Prato, M. The Covalent Functionalization of Graphene on Substrates. Angew. Chem. Int. Ed. 2015, DOI: 10.1002/anie.201501473.

28. Marchesan, S.; Bosi, S.; Prato, M. Carbon Nanotubes for Organ Regeneration: An Electrifying Performance. 2015, submitted.

29. Martinelli, V.; Cellot, G.; Toma, F. M.; Long, C. S.; Caldwell, J. H.; Zentilin, L.; Giacca, M.; Turco, A.; Prato, M.; Ballerini, L.; et al. Carbon Nanotubes Instruct Physiological Growth and Functionally Mature Syncytia: Nongenetic Engineering of Cardiac Myocytes. ACS Nano 2013, 7, 5746-5756.

30. Zhou, J.; Chen, J.; Sun, H.; Qiu, X.; Mou, Y.; Liu, Z.; Zhao, Y.; Li, X.; Han, Y.; Duan, C.; et al. Engineering the Heart: Evaluation of Conductive Nanomaterials for Improving Implant Integration and Cardiac Function. Sci. Rep. 2014, 4, 3733.

31. Kharaziha, M.; Shin, S. R.; Nikkhah, M.; Topkaya, S. N.; Masoumi, N.; Annabi, N.; Dokmeci, M. R.; Khademhosseini, A. Tough and Flexible CNT-Polymeric Hybrid Scaffolds for Engineering Cardiac Constructs. Biomaterials 2014, 35, 7346-7354.

32. Li, X.; Zhou, J.; Liu, Z.; Chen, J.; Lü, S.; Sun, H.; Li, J.; Lin, Q.; Yang, B.; Duan, C.; et al. A PNIPAAmBased Thermosensitive Hydrogel Containing SWCNTs for Stem Cell Transplantation in Myocardial Repair. Biomaterials 2014, 35, 5679-5688.

33. Pok, S.; Vitale, F.; Eichmann, S. L.; Benavides, O. M.; Pasquali, M.; Jacot, J. G. Biocompatible Carbon Nanotube-Chitosan Scaffold Matching the Electrical Conductivity of the Heart. ACS Nano 2014, 8, 9822-9832.

34. Shin, S. R.; Jung, S. M.; Zalabany, M.; Kim, K.; Zorlutuna, P.; Kim, S. B.; Nikkhah, M.; Khabiry, M.; Azize, M.; Kong, J.; et al. Carbon-Nanotube-Embedded Hydrogel Sheets for Engineering Cardiac Constructs and Bioactuators. ACS Nano 2013, 7, 2369-2380.

35. Shin, S. R.; Aghaei-Ghareh-Bolagh, B.; Gao, X.; Nikkhah, M.; Jung, S. M.; Dolatshahi-Pirouz, A.; Kim, S. B.; Kim, S. M.; Dokmeci, M. R.; Tang, X. S.; et al. Layer-by-Layer Assembly of 3D Tissue Constructs with Functionalized Graphene. Adv. Funct. Mater. 2014, 24, 6136-6144.

36. Fabbro, A.; Bosi, S.; Ballerini, L.; Prato, M. Carbon Nanotubes: Artificial Nanomaterials to Engineer Single Neurons and Neuronal Networks. ACS Chem. Neurosci. 2012, 3, 611-618.

37. Bosi, S.; Rauti, R.; Laishram, J.; Turco, A.; Lonardoni, D.; Nieus, T.; Prato, M.; Scaini, D.; Ballerini, L. From 2D to 3D: Novel Nanostructured Scaffolds To Investigate Signalling in Reconstructed Neuronal Networks. Sci. Rep. 2015, 5, 9562.

38. Fabbro, A.; Sucapane, A.; Toma, F. M.; Calura, E.; Rizzetto, L.; Carrieri, C.; Roncaglia, P.; Martinelli, V.; Scaini, D.; Masten, L.; Turco, A.; et al. Adhesion to Carbon Nanotube Conductive Scaffolds Forces Action-Potential Appearance in Immature Rat Spinal Neurons. PLoS One 2013, 8 , e73621.

39. Aldinucci, A.; Turco, A.; Biagioli, T.; Toma, F. M.; Bani, D.; Guasti, D.; Manuelli, C.; Rizzetto, L.; Cavalieri, D.; Massacesi, L.; et al. Carbon Nanotube Scaffolds Instruct Human Dendritic Cells: Modulating Immune Responses by Contacts at the Nanoscale. Nano Lett. 2013, 13, 6098-105.

40. Landers, J.; Turner, J. T.; Heden, G.; Carlson, A. L.; Bennett, N. K.; Moghe, P. V.; Neimark, A. V. Carbon Nanotube Composites as Multifunctional Substrates for In Situ Actuation of Differentiation of Human Neural Stem Cells. Adv. Healthc. Mater. 2014, 3, 1745-1752. 
41. Fabbro, A.; Villari, A.; Laishram, J.; Scaini, D.; Toma, F. M.; Turco, A.; Prato, M.; Ballerini, L. Spinal Cord Explants Use Carbon Nanotube Interfaces To Enhance Neurite Outgrowth and To Fortify Synaptic Inputs. ACS Nano 2012, 6, 2041-2055.

42. Cellot, G.; Cilia, E.; Cipollone, S.; Rancic, V.; Sucapane, A.; Giordani, S.; Gambazzi, L.; Markram, H.; Grandolfo, M.; Scaini, D.; et al. Carbon Nanotubes Might Improve Neuronal Performance by Favouring Electrical Shortcuts. Nat. Nanotechnol. 2009, 4, 126-133.

43. Bardi, G.; Nunes, A.; Gherardini, L.; Bates, K.; Al-Jamal, K. T.; Gaillard, C.; Prato, M.; Bianco, A.; Pizzorusso, T.; Kostarelos, K. Functionalized Carbon Nanotubes in the Brain: Cellular Internalization and Neuroinflammatory Responses. PLoS One 2013, 8, e80964/1-e80964/10.

44. Bosi, S.; Fabbro, A.; Cantarutti, C.; Mihajlovic, M.; Ballerini, L.; Prato, M. Carbon Based Substrates for Interfacing Neurons: Comparing Pristine with Functionalized Carbon Nanotubes Effects on Cultured Neuronal Networks. Carbon 2016, 97, 87-91.

45. Fuchsberger, K.; Le Goff, A.; Gambazzi, L.; Toma, F. M.; Goldoni, A.; Giugliano, M.; Stelzle, M.; Prato, M. Multiwalled Carbon-Nanotube-Functionalized Microelectrode Arrays Fabricated by Microcontact Printing: Platform for Studying Chemical and Electrical Neuronal Signaling. Small 2011, 7 , 524-530.

46. Kotov, N. A.; Winter, J. O.; Clements, I. P.; Jan, E.; Timko, B. P.; Campidelli, S.; Pathak, S.; Mazzatenta, A.; Lieber, C. M.; Prato, M.; et al. Nanomaterials for Neural Interfaces. Adv. Mater. 2009, 21, 3970-4004.

47. Gaillard, C.; Cellot, G.; Li, S.; Toma, F. M.; Dumortier, H.; Spalluto, G.; Cacciari, B.; Prato, M.; Ballerini, L.; Bianco, A. Carbon Nanotubes Carrying Cell-Adhesion Peptides do not Interfere with Neuronal Functionality. Adv. Mater. 2009, 21, 2903-2908.

48. Nayak, T. R.; Andersen, H.; Makam, V. S.; Khaw, C.; Bae, S.; Xu, X.; Ee, P.-L. R.; Ahn, J.-H.; Hong, B. H.; Pastorin, G.; et al. Graphene for Controlled and Accelerated Osteogenic Differentiation of Human Mesenchymal Stem Cells. ACS Nano 2011, 5, 4670-4678.

49. Shah, S.; Yin, P. T.; Uehara, T. M.; Chueng, S.-T. D.; Yang, L.; Lee, K.-B. Guiding Stem Cell Differentiation into Oligodendrocytes Using Graphene-Nanofiber Hybrid Scaffolds. Adv. Mater. 2014, 26, 3673-3680.

50. Lee, W. C.; Lim, C. H. Y. X.; Shi, H.; Tang, L. A. L.; Wang, Y.; Lim, C. T.; Loh, K. P. Origin of Enhanced Stem Cell Growth and Differentiation on Graphene and Graphene Oxide. ACS Nano 2011, 5 , 7334-7341.

51. Marchesan, S.; Prato, M. Under the Lens: Carbon Nanotube and Protein Interaction at the Nanoscale. Chem. Commun. 2015, 51, 4347-4359.

52. Lorenzoni, M.; Brandi, F.; Dante, S.; Giugni, A.; Torre, B. Simple and Effective Graphene Laser Processing for Neuron Patterning Application. Sci. Rep. 2013, 3, 1954.

53. Kumar, S.; Raj, S.; Kolanthai, E.; Sood, A. K.; Sampath, S.; Chatterjee, K. Chemical Functionalization of Graphene To Augment Stem Cell Osteogenesis and Inhibit Biofilm Formation on Polymer Composites for Orthopedic Applications. ACS Appl. Mater. Interfaces 2015, 7, 3237-3252.

54. Melchionna, M.; Bonchio, M.; Paolucci, F.; Prato, M.; Fornasiero, P. Catalysis-Material Crosstalk at Tailored Nano-Carbon Interfaces. Top. Curr. Chem. 2014, 348, 139-180.

55. Cargnello, M.; Grzelczak, M.; Rodriguez-Gonzalez, B.; Syrgiannis, Z.; Bakhmutsky, K.; La Parola, V.; Liz-Marzan, L. M.; Gorte, R. J.; Prato, M.; Fornasiero, P. Multiwalled Carbon Nanotubes Drive the Activity of Metal@Oxide Core-Shell Catalysts in Modular Nanocomposites. J. Am. Chem. Soc. 2012, 134, 11760-11766.

56. Casillas, R.; Lodermeyer, F.; Costa, R. D.; Prato, M.; Guldi, D. M. Substituting $\mathrm{TiCl}_{4}-\mathrm{Carbon}$ Nanohorn Interfaces for Dye-Sensitized Solar Cells. Adv. Energy Mater. 2014, 4, 1301577.

57. Li, Y.; Wang, H.; Xie, L.; Liang, Y.; Hong, G.; Dai, H. MoS 2 Nanoparticles Grown on Graphene: An Advanced Catalyst for the Hydrogen Evolution Reaction. J. Am. Chem. Soc. 2011, 133, 7296-7299. 
58. Tran, P. D.; Le Goff, A.; Heidkamp, J.; Jousselme, B.; Guillet, N.; Palacin, S.; Dau, H.; Fontecave, M.; Artero, V. Noncovalent Modification of Carbon Nanotubes with Pyrene-Functionalized Nickel Complexes: Carbon Monoxide Tolerant Catalysts for Hydrogen Evolution and Uptake. Angew. Chem. Int. Ed. 2011, 50, 1371-1374.

59. Li, F.; Zhang, B.; Li, X.; Jiang, Y.; Chen, L.; Li, Y.; Sun, L. Highly Efficient Oxidation of Water by a Molecular Catalyst Immobilized on Carbon Nanotubes. Angew. Chem. Int. Ed. 2011, 50, 12276-12279.

60. Yano, J.; Kern, J.; Sauer, K.; Latimer, M. J.; Pushkar, Y.; Biesiadka, J.; Loll, B.; Saenger, W.; Messinger, J.; Zouni, A.; et al. Where Water Is Oxidized to Dioxygen: Structure of the Photosynthetic $\mathrm{Mn}_{4} \mathrm{Ca}$ Cluster. Science 2006, 314, 821-825.

61. Toma, F. M.; Sartorel, A.; Iurlo, M.; Carraro, M.; Parisse, P.; Maccato, C.; Rapino, S.; Gonzalez, B. R.; Amenitsch, H.; Da Ros, T.; et al. Efficient Water Oxidation at Carbon Nanotube-Polyoxometalate Electrocatalytic Interfaces. Nat. Chem. 2010, 2, 826-831.

62. Toma, F. M.; Sartorel, A.; Iurlo, M.; Carraro, M.; Rapino, S.; Hoober-Burkhardt, L.; Da Ros, T.; Marcaccio, M.; Scorrano, G.; Paolucci, F.; et al. Tailored Functionalization of Carbon Nanotubes for Electrocatalytic Water Splitting and Sustainable Energy Applications. ChemSusChem 2011, 4, 1447-1451. 63. Quintana, M.; López, A. M.; Rapino, S.; Toma, F. M.; lurlo, M.; Carraro, M.; Sartorel, A.; Maccato, C.; Ke, X.; Bittencourt, C.; et al. Knitting the Catalytic Pattern of Artificial Photosynthesis to a Hybrid Graphene Nanotexture. ACS Nano 2013, 7, 811-817.

64. Guo, S.; Sun, S. FePt Nanoparticles Assembled on Graphene as Enhanced Catalyst for Oxygen Reduction Reaction. J. Am. Chem. Soc. 2012, 134, 2492-2495.

65. Wei, Z. D.; Yan, C.; Tan, Y.; Li, L.; Sun, C. X.; Shao, Z. G.; Shen, P. K.; Dong, H. W. Spontaneous Reduction of Pt(IV) onto the Sidewalls of Functionalized Multiwalled Carbon Nanotubes as Catalysts for Oxygen Reduction Reaction in PEMFCs. J. Phys. Chem. C 2008, 112, 2671-2677.

66. Liang, Y.; Li, Y.; Wang, H.; Zhou, J.; Wang, J.; Regier, T.; Dai, H. $\mathrm{CO}_{3} \mathrm{O}_{4}$ Nanocrystals on Graphene as a Synergistic Catalyst for Oxygen Reduction Reaction. Nat. Mater. 2011, 10, 780-786.

67. Liang, Y.; Wang, H.; Diao, P.; Chang, W.; Hong, G.; Li, Y.; Gong, M.; Xie, L.; Zhou, J.; Wang, J.; et al. Oxygen Reduction Electrocatalyst Based on Strongly Coupled Cobalt Oxide Nanocrystals and Carbon Nanotubes. J. Am. Chem. Soc. 2012, 134, 15849-15857.

68. Pan, X.; Bao, X. The Effects of Confinement inside Carbon Nanotubes on Catalysis. Acc. Chem. Res. 2011, 44, 553-562.

69. Deng, D.; Yu, L.; Chen, X.; Wang, G.; Jin, L.; Pan, X.; Deng, J.; Sun, G.; Bao, X. Iron Encapsulated within Pod-like Carbon Nanotubes for Oxygen Reduction Reaction. Angew. Chem. Int. Ed. 2013, 52, 371375.

70. Bracamonte, M. V.; Melchionna, M.; Stopin, A.; Giulani, A.; Tavagnacco, C.; Garcia, Y.; Fornasiero , P.; Bonifazi , D.; Prato, M. Carboxylated, Fe-Filled Multiwalled Carbon Nanotubes as Versatile Catalysts for $\mathrm{O}_{2}$ Reduction and $\mathrm{H}_{2}$ Evolution Reactions at Physiological pH. Chem. Eur. J. 2015, DOI: 10.1002/chem.201501144.

Suggested Pull Quotes

Carbon nanotubes and graphene molecularly and electronically bridge in a manner that is useful across many disciplines of science, from medicine to energy. 
At this pace of development, and in parallel with the enormous advances in the scaled-up preparation of high-quality carbon nanostructures (CNS), we anticipate a new generation of solar fuel cells with CNS as essential components. 